\title{
... hat ein hebraisch zettel dabey. Der Umgang mit jüdisch-christlichen Geschäfts- urkunden im spätmittelalterlichen Österreich*
}

\begin{abstract}
Die Überlieferung spätmittelalterlicher Urkunden zu jüdisch-christlichen Geschäftskontakten, vor allem aus dem Bereich des Kreditwesens, ist in Österreich ungewöhnlich reichhaltig. Die Quellenlage erlaubt Untersuchungen zum Umgang mit den Urkunden als physische Objekte, zu Aufbewahrung, Verlust oder bewusster Zerstörung sowie zum Einsatz der Urkunden im spannungsgeladenen Feld jüdischchristlicher Interaktion. Gerade das Geldgeschäft mit seinen potentiellen Konfliktfeldern, die noch zusätzlich durch landesfürstliche Eingriffe verschärft werden konnten, machte für beide Seiten rechtsverbindliche Dokumente essentiell, wobei man sich weitgehend an der christlichen Beurkundungspraxis orientierte, aber von christlicher Seite auch jüdische Traditionen wie die Beglaubigung durch Unterschrift akzeptierte. Allerdings führte dies dazu, dass neben dem Geld und den Wertgegenständen der Juden auch ihre offenen Schuldbriefe die Begehrlichkeiten christlicher Verfolger weckten, sodass im Zug der Zerstörung der österreichischen Judengemeinden 1420/21 auch die jüdischen Geschäftsurkunden zu einem Teil der Beute wurden. Dass so viele dieser Urkunden die Präsenz der Juden im mittelalterlichen Österreich überdauerten, während der Großteil des innerjüdischen Schriftguts verloren ging, wirft trotz der, geteilten Formeln' der Urkundenpraxis ein bezeichnendes Licht auf die prekäre Position der Juden und die vom wirtschaftlichen Interesse der christlichen Obrigkeit geprägte Rolle, auf die sie reduziert wurden.
\end{abstract}

Im Jahr 1339 stellte der Wiener Jude Schalaun, Sohn des Gutman, mit seiner Frau Ester eine Urkunde über den Verkauf eines Weingartens an einen Gefolgsmann des österreichischen Herzogs Albrecht II. aus. Die Corroboratio der Urkunde stellt neben der Ankündigung der Siegler allgemein fest: Und daz diser chauf fuerbaz also staet und unzerbrochen beleibe [...] dar umbe so geben wir in disen brief zu einem warn urchunde und zu einer ewigen vestunge diser sache. ${ }^{1}$ Die Aussteller bedienten sich damit einer Formel, wie sie sich tausendfach in spätmittelalterlichen Geschäftsbrie-

\footnotetext{
* Dieser Beitrag basiert auf Forschungsergebnissen aus dem vom österreichischen Forschungsfonds (FWF) finanzierten Projekt „Regesten zur Geschichte der Juden in Ostösterreich 1387-1404“ (P 24404) und den Vorgängerprojekten P 15638, P 18453 und P 21236.

1 Wiener Stadt- und Landesarchiv, Bürgerspitalsurkunde Nr. 85. Regest bei: Eveline BRUGGER u. Birgit WIEDL, Regesten zur Geschichte der Juden in Österreich im Mittelalter, Bd. 2: 1339-1365, Innsbruck, Wien, Bozen 2010, S. 10, Nr. 459.
} 
fen $^{2}$ findet - im ausschließlich christlichen Umfeld ebenso wie in Urkunden, die aus christlich-jüdischen Geschäftskontakten hervorgingen. Letztere stellen aufgrund der vergleichsweise dichten Überlieferungslage die wichtigste Quellengattung für das jüdische Wirtschaftsleben im österreichischen Spätmittelalter dar, während sich die ab dem 14. Jahrhundert von christlichen Obrigkeiten zur zusätzlichen Absicherung von Pfand- und Kreditgeschäften angelegten Judenbücher nur in seltenen Fällen erhalten haben. ${ }^{3}$

Gerade im geschäftlichen Umfeld war es für alle Beteiligten wichtig, klare Beweismittel für den ordnungsgemäßen Abschluss einer Transaktion zur Verfügung zu haben. Bei Geschäftsabschlüssen zwischen Christen und Juden kam noch hinzu, dass die getroffenen Vereinbarungen für das Rechtsverständnis beider Seiten verbindlich sein mussten; es war daher nötig, einen gemeinsamen Nenner zu finden, der sich - wenig überraschend - zum größten Teil an der christlichen Beurkundungspraxis orientierte. Die Etablierung der jüdischen Ansiedlung im Herzogtum Österreich erfolgte im 13. Jahrhundert ${ }^{4}$ und fällt mit der Zeit zusammen, in der bei Rechtsgeschäften zunehmend auf Schriftlichkeit Wert gelegt wurde. Zunächst im Adel, später dann auch im bürgerlichen Umfeld, entwickelte sich ein privaturkundliches Formular, das mit nur geringen Adaptierungen auch im jüdisch-christlichen Geschäftsverkehr zum Einsatz kam - egal ob der Aussteller der Urkunde nun Christ oder Jude war. ${ }^{5}$

2 Der Begriff „Geschäftsurkunde“ bzw. „-brief“ wird in diesem Beitrag im Sinne von „Privaturkunde, die aus einer wirtschaftlichen Transaktion hervorgegangen ist“ verwendet und folgt nicht der von Oswald REDLICH eingeführten Gleichsetzung von „Geschäftsurkunde“ mit „Carta“ (= dispositive Urkunde) im Gegensatz zur streng davon unterschiedenen „Notitia = Beweisurkunde“. Oswald REDLICH, Die Privaturkunden des Mittelalters. Urkundenlehre, Bd. 3, hrsg. v. Wilhelm ERBEN, Ludwig SCHMITZ-KALLENBERG u. Oswald REDLICH (Handbuch der mittelalterlichen und neueren Geschichte, Abt. IV: Hilfswissenschaften und Altertümer), München, Berlin 1911, S. 4-8, 115-124.

$3 \mathrm{Zu}$ den Geschäftsurkunden und deren Erschließung im Rahmen des laufenden Forschungsprojekts „Regesten zur Geschichte der Juden in Österreich“ (http://injoest.ac.at/projekte/laufend/ mittelalterliche_judenurkunden/; einges. 7.6.2014) vgl. Eveline BRUGGER u. Birgit WIEDL, ...und ander frume leute genuch, paide christen und juden. Quellen zur christlich-jüdischen Interaktion im Spätmittelalter, in: Rolf KiEssling u. a. (Hgg.), Räume und Wege. Jüdische Geschichte im Alten Reich 1300-1800 (Colloquia Augustana 25), Berlin 2007, S. 285-305, hier S. 290-293, 301-305. Zu den Judenbüchern vgl. Thomas PETER, Judenbücher als Quellengattung und die Znaimer Judenbücher. Typologie und Forschungsstand, in: ebd. S. 307-331, hier S. 307-319; Klaus LoHRMANN, Judenrecht und Judenpolitik im mittelalterlichen Österreich, Wien, Köln 1990, S. $158 f$.

4 Eveline BRUgGER, Von der Ansiedlung bis zur Vertreibung - Juden in Österreich im Mittelalter, in: DIES. u. a., Geschichte der Juden in Österreich, 2. Aufl. Wien 2013, S. 123-228, hier S. 126-129; LoHRMANN (Anm. 3), S. 46-53.

5 Roman ZehetmaYer, Urkunde und Adel. Ein Beitrag zur Geschichte der Schriftlichkeit im Südosten des Reichs vom 11. bis zum frühen 14. Jahrhundert (Veröffentlichungen des Instituts für Österreichische Geschichtsforschung 53), Wien, München 2010, S. 263-265. Selbst die wenigen erhaltenen hebräischen Urkunden folgen weitgehend demselben Formular, vgl. BRUGGER u. WIEDL (Anm. 3), 
Über Form und Rechtsinhalt dieser Urkunden liegen mehrere Untersuchungen jüngeren Datums vor. ${ }^{6}$ An dieser Stelle soll das Hauptaugenmerk daher auf den praktischen Umgang mit den Urkunden im Lauf und nach Beendigung der darin festgehaltenen Geschäftsgänge gelegt werden, sowie auf die Rolle, die sie in der - friedlichen oder gewaltsamen - jüdisch-christlichen Interaktion spielten.

Vorausschickend muss festgestellt werden, dass das Kreditgeschäft nicht nur in den Quellen zur jüdischen Geschichte Österreichs im Allgemeinen, sondern vor allem in den Geschäftsurkunden überproportional stark repräsentiert ist. ${ }^{7}$ Die Annahme, dies sei auf eine ausschließliche Beschränkung der jüdischen Tätigkeit auf den Geldverleih zurückzuführen, ist längst widerlegt, ${ }^{8}$ ebenso wie die Vermutung, dass Juden und Christen nur im Rahmen von Kreditgeschäften in Kontakt gekommen wären. ${ }^{9}$ Trotzdem ist die aus der Ungleichgewichtung der Quellen resultierende Verzerrung ein Faktor, der bei daraus gezogenen Schlussfolgerungen immer einberechnet werden muss. Dazu kommt noch die Tatsache, dass eine schriftliche Fixierung früher und häufiger für hohe Darlehen, die naturgemäß nur zwischen den Eliten beider Seiten vergeben bzw. aufgenommen werden konnten, für nötig erachtet wurde, als dies für das kleine, alltägliche Pfandgeschäft der Fall war. ${ }^{10}$ Zuletzt ist auch noch die Überlieferungssituation in Betracht zu ziehen, denn Geschäftsurkunden mit jüdischer

S. 294; Martha KeIL, Gemeinde und Kultur - die mittelalterlichen Grundlagen jüdischen Lebens in Österreich, in: BRUGGER u. a. (Anm. 4), S. 15-122, hier S. 33.

6 Wilhelm WADL, Geschichte der Juden in Kärnten im Mittelalter. Mit einem Ausblick bis zum Jahre 1867 (Das Kärntner Landesarchiv 9), 3. Aufl. Klagenfurt 2009, S. 36-38; LoHRMANN (Anm. 3), S. 171-182; BRUGGER u. WIEDL (Anm. 3), S. 285-289; künftig auch: Eveline BRUGGER, Urkunden zum jüdischen Kreditgeschäft im mittelalterlichen Österreich (Forschungen zur Geschichte der Juden. Schriftenreihe der Gesellschaft zur Erforschung der Juden e. V. und des Arye Maimon-Instituts für Geschichte der Juden [erscheint 2014]).

7 Eveline BRUgGer, Do musten da hin zue den iuden varn - die Rolle(n) jüdischer Geldgeber im spätmittelalterlichen Österreich, in: DIES. u. Birgit WIEDL (Hgg.), Ein Thema - zwei Perspektiven. Juden und Christen in Mittelalter und Frühneuzeit, Innsbruck, Wien u. Bozen 2007, S. 122-138, hier S. 123.

8 Michael TocH, Zur wirtschaftlichen Lage und Tätigkeit der Juden im deutschen Sprachraum des Spätmittelalters, in: Rolf KIEssuING (Hg.), Judengemeinden in Schwaben im Kontext des Alten Reiches, Berlin 1995, S. 39-50. Zu Österreich vgl. BRUGGER (Anm. 4), S. 166-168.

9 Aus der umfangreichen Literatur vgl. z. B. Martha KeIL, Kulicht schmalz und eisen gaffel - Alltag und Repräsentation bei Juden und Christen im Spätmittelalter, in: Aschkenas 14, 1 (2004), S. 51-81; Markus WENNIngER, Von der Integration zur Segregation. Die Entwicklung deutscher Judenviertel im Mittelalter, in: Eveline BRUgGeR u. Birgit WiEdL (Hgg.), Ein Thema - zwei Perspektiven. Juden und Christen in Mittelalter und Frühneuzeit, Innsbruck, Wien, Bozen 2007, S. 195-217; Birgit WiEDL, Jews and the City: Parameters of Urban Jewish Life in Late Medieval Austria, in: Albrecht Classen (Hg.), Urban Space in the Middle Ages and the Early Modern Age (Fundamentals of Medieval and Early Modern Culture 4), Berlin 2009, S. 273-308; Robert CHAZAN, Reassessing Jewish Life in Medieval Europe, Cambridge u. a. 2010, S. 182-189.

10 Brugger u. Wiedl (Anm. 3), S. $290 f$. 
Beteiligung sind fast immer über die Aufbewahrung beim christlichen Geschäftspartner auf uns gekommen, worauf noch einzugehen sein wird. ${ }^{11}$

Gerade beim Kreditgeschäft über hohe Summen bzw. gegen wertvolle Pfänder war aufgrund der damit verbundenen Risiken die rechtsverbindliche schriftliche Absicherung sowohl für den Schuldner als auch für den Gläubiger von Bedeutung. Der Geldverleih warf außerdem Problemfelder auf, die nicht nur mit der Dokumentation eines Geschäftsverlaufs, sondern auch mit dessen Ende zu tun hatten. Im Gegensatz zu einem auf dauerhafte Gültigkeit angelegten Geschäft wie dem eingangs zitierten Weingartenverkauf wurden Kredite ja in der Annahme aufgenommen, dass sie zu einem bestimmten Zeitpunkt zurückgezahlt werden würden; ${ }^{12}$ sobald diese Rückzahlung erfolgte, hatte der Schuldner ein verständliches Interesse daran, dass die Gültigkeit des ursprünglichen Schuld- oder Pfandbriefes rechtsgültig widerrufen wurde. Der reguläre Endpunkt eines Kreditgeschäfts war daher entweder der Quittbrief, in dem der Gläubiger die vollständige Begleichung der Schuld bestätigte, oder aber im Falle der Nichtbezahlung die urkundliche Übertragung des verfallenen Pfandes in den Besitz des Gläubigers. Gängiger Bestandteil solcher Urkunden war die formelhafte Ungültigkeitserklärung aller bis dahin ausgestellten Schuldbriefe, die nach dem üblichen Sprachgebrauch für „,tot und kraftlos“ erklärt wurden bzw. „keine Gewalt mehr haben“ sollten.

Allerdings kam nur ein Teil der Kreditgeschäfte - und dies gilt im Besonderen, wenn auch nicht ausschließlich für jüdische Kreditgeschäfte - zu einem solch regulären Endpunkt. Die in Österreich seit der ersten Hälfte des 14. Jahrhunderts auftretenden herzoglichen Tötbriefe gingen einen entscheidenden Schritt weiter: Es waren jetzt nicht mehr die involvierten Geschäftspartner selbst, die die Ungültigkeit einer jüdischen Schuld- oder Pfandurkunde bestätigten, sondern der Landesfürst, der seine Position als Herr der Juden seines Landes dazu nutzte, Darlehen seiner jüdischen „Kammerknechte“ eigenmächtig für nichtig zu erklären. ${ }^{13}$

11 Mittelalterliche Urkunden zu jüdisch-christlichen Geschäftskontakten in Österreich finden sich heute vor allem in den Urkundensammlungen zahlreicher Klosterarchive (die zum Teil später an staatliche Archive transferiert wurden), adeliger Privatarchive (die, sofern sie nicht zeitnah an ein Kloster übergeben wurden, heute ebenfalls häufig in staatlichen Archiven oder Archiven der Bundesländer zu finden sind) sowie der Archive jener Städte, in denen jüdische Gemeinden bestanden. Vgl. das Material bei Eveline BRUGGER u. Birgit WIEDL, Regesten zur Geschichte der Juden in Österreich im Mittelalter, Bd. 1: Von den Anfängen bis 1338, Innsbruck, Wien, Bozen 2005, S. 15-351; DIEs. (Anm. 1), S. 9-337.

12 Arnold Esch, Überlieferungs-Chance und Überlieferungs-Zufall als methodisches Problem des Historikers, in: Historische Zeitschrift 240 (1985), S. 529-570, hier S. 535f.

13 Nutznießer herrscherlicher Tötbriefe waren meist Adelige, die der Herzog durch die Stundung oder sogar den völligen Erlass ihrer Schulden bei den herzoglichen Juden zu fördern wünschte - sei es, um sich ihrer Loyalität zu versichern, sei es, um eigene Schulden bei adeligen Gefolgsleuten auf diese Weise zu begleichen. Das Mittel der Schuldentötung konnte auch als Druckmittel oder Strafmaßnahme gegen jüdische Untertanen angewendet werden, wenn diese zum Beispiel ohne Erlaubnis des 
Die Beendigung eines Kreditgeschäfts - ob diese nun regulär oder durch herrscherlichen Eingriff erfolgte - warf die Frage auf, wie mit den erledigten Urkunden umgegangen werden sollte. Häufig wurde bereits bei Abschluss des Geschäfts vereinbart, dass der Kreditnehmer die Schuldurkunde nach Begleichung der Schuld zurückerhalten würde. In vielen Klöstern, aber auch in den Archiven mancher Adelsfamilien wurden diese erledigten Schuldbriefe dauerhaft aufbewahrt, worauf die Tatsache zurückzuführen ist, dass sich eine große Anzahl jüdischer Geschäftsurkunden erhalten hat, während die meisten hebräischen Bücher und Handschriften, die im Besitz der Juden verblieben, im Zuge der Verfolgungen und Vertreibungen des Spätmittelalters verloren gingen. ${ }^{14}$

Zwar war auch die absichtliche Zerstörung oder zumindest sichtbare Beschädigung von Schuldurkunden nicht selten, doch war diese meist die Folge des regulären Endes eines Kreditgeschäfts. Es war - nicht nur bei Geschäften mit jüdischer Involvierung - gängig, erledigte Schuldurkunden durch Einschnitte zu kassieren und auf diese Weise sichtbar als nicht mehr gültig zu kennzeichnen. Kassationsschnitte finden sich ab dem 14. Jahrhundert an zahlreichen jüdischen Schuldbriefen, aber auch an Bürgschaftsurkunden und ähnlichen Dokumenten aus dem Umfeld eines Kreditgeschäfts. ${ }^{15}$

Nicht immer wurde es in der Folge als nötig erachtet, den Beleg eines erledigten Darlehens auf Dauer aufzubewahren. Aus der Urkunde wurde damit ein Stück Rohmaterial, das möglichst sinnvoll weiterverwendet werden sollte. So waren z. B. die Mönche des niederösterreichischen Zisterzienserklosters Zwettl äußerst sparsam im Umgang mit dem teuren Pergament und verarbeiteten nicht mehr benötigte Schuldbzw. Pfandurkunden zu Siegeltaschen für diejenigen Stücke, die als archivwürdig

Landesherrn dessen Territorium verließen. Eveline BRUGGER, „So sollen die brief ab und tod sein.“ Landesfürstliche Judenschuldentilgungen im Österreich des 14. Jahrhunderts, in: Eveline BRUGGER u. Birgit WIEDL (Hgg.), Jüdisches Geldgeschäft im Mittelalter (Aschkenas 20, 2), Berlin, Boston 2012, S. 329-341. Zur Kammerknechtschaft vgl. David ABulafiA, Der König und die Juden - Juden im Dienst des Herrschers, in: Christoph CLusE (Hg.), Europas Juden im Mittelalter. Beiträge des internationalen Symposiums in Speyer vom 20. bis 25. Oktober 2002, Trier 2004, S. 60-71; Michael TocH, Die Juden im mittelalterlichen Reich (Enzyklopädie deutscher Geschichte 44), 2. Aufl. München 2003, S. 48, 104-106; Alfred HAVERKAMP, „Kammerknechtschaft“ und „Bürgerstatus“ der Juden diesseits und jenseits der Alpen während des späten Mittelalters, in: Michael Brenner u. Sabine UlLmann (Hgg.), Die Juden in Schwaben, München 2013, S. 11-40, hier S. 11-20, 30-34.

14 Martha KeIL, Heilige Worte - Schriften des Abscheus. Der Umgang mit Büchern als Paradigma des jüdisch-christlichen Spannungsverhältnisses, in: Karl BRUnNER u. Gerhard JARITz (Hgg.), Text als Realie. Internationaler Kongress Krems an der Donau, 3. bis 6. Oktober 2000 (Österreichische Akademie der Wissenschaften, Philosophisch-historische Klasse, Sitzungsberichte 704 = Veröffentlichungen des Instituts für Realienkunde des Mittelalters und der Frühen Neuzeit 18), Wien 2003, S. 49-61, hier S. 59f.

15 Vgl. z. B. Wien, Haus-, Hof- und Staatsarchiv, Allgemeine Urkundenreihe 1362 III 31. Regest bei: BRUgGer u. WiEdL (Anm. 1), S. 274, Nr. 1017. 
betrachtet wurden. Auf diese Weise überlieferten sie unabsichtlich auch die Namen einiger sonst nicht bekannter jüdischer Geldleiher aus dem Zwettler Raum. ${ }^{16}$ Gerade für die sonst nur schwer quellenmäßig fassbare jüdische Präsenz in kleinen, ländlichen Siedlungen spielen solche Zufallsüberlieferungen eine große Rolle. ${ }^{17}$

Eine andere Möglichkeit des ,Recyclings‘ stellten die Einbände oder Vorsatzblätter von Handschriften dar. Erledigte jüdische Schuldurkunden oder deren Fragmente finden sich in den Codices mehrerer österreichischer Bibliotheken und Archive. ${ }^{18}$ Bucheinbände sind auch häufige Fundorte von Fragmenten aus hebräischen Handschriften; ${ }^{19}$ allerdings waren diese wie erwähnt meist auf gewaltsamem Weg in den Besitz des christlichen Skriptoriums geraten, während bei der Verwendung von Urkunden in diesem Zusammenhang eher von einem regulären Ende des darin dokumentierten Geschäfts ausgegangen werden kann.

Umgekehrt trat auch immer wieder der Fall ein, dass Urkunden in Verlust gerieten, bevor das entsprechende Geschäft beendet war. Besonders problematisch war dies, wenn nicht mehr alle der ursprünglich Beteiligten für die Rekonstruktion des

16 Zwettl, Stiftsarchiv, Urkunden 1315 IV 4, 1316, 1317 VI 11, 1321 I 21?, (um) 1326, (um) 1326, 1325-1329 vor III 12. Rekonstruktion der Texte bei: BRugger u. WiedL (Anm. 11), S. 190f., Nr. 196, S. 196, Nr. 206, S. 198f., Nr. 210, S. 213f., Nr. 234, S. 237-239, Nr. 275f., S. 265f., Nr. 316. Vgl. Birgit WiEDL, Die Zwettler Siegeltaschen - ein historisches Puzzle, in: Institut für jüdische Geschichte Österreichs (Hg.), Zwischen den Zeilen. 20 Jahre Institut für jüdische Geschichte Österreichs, Wien 2008, S. 32-38; DIES., „Lazarus and Abraham, our Jews of Eggenburg“: Jews in the Austrian Countryside in the Fourteenth Century, in: Albrecht Classen (Hg.), Rural Space in the Middle Ages and Early Modern Age. The Spatial Turn in Premodern Studies (Fundamentals of Medieval and Early Modern Culture 9), Berlin, Boston 2012, S. 639-672, hier S. 646; Eveline BRUGGER, „...daz wier schullen gelten Abraham dem juden von Zwetel...“ Mittelalterliche Spuren jüdischen Lebens im Waldviertel, in: Friedel MoLL, Jüdisches Leben in Zwettl. Koexistenz und Verfolgung, vom Mittelalter bis ins 20. Jahrhundert (Zwettler Zeitzeichen 13), Zwettl 2009, S. 8-15, hier S. 9f.

17 Vgl. allgemein EsCH (Anm. 12), S. 544-548. Zur aufgrund der Quellenarmut lange unterschätzten jüdischen Siedlung auf dem Land vgl. WiEdL (Anm. 16); Rainer BARzEN, Benei haKefarim - die Leute aus den Dörfern: Zur jüdischen Siedlung auf dem Lande in Aschkenas und Zarfat im hohen und späteren Mittelalter, in: Frank G. Hirschmann u. Gerd Mentgen (Hgg.), Campana pulsante convocati. Festschrift anläßlich der Emeritierung von Prof. Dr. Alfred Haverkamp, Trier 2005, S. 21-37; DERS., Ländliche jüdische Siedlungen und Niederlassungen in Aschkenas. Vom Hochmittelalter bis ins 16. Jahrhundert. Typologie, Struktur und Vernetzung, in: Aschkenas 21, 1/2 (2011), S. 5-35, hier S. 5-28.

18 Vgl. z. B. Klagenfurt, Kärntner Landesarchiv, Urkunde A 512 (1382 Juni 2): die Urkunde des Juden Mosche aus Voitsberg über den Verkauf eines ihm als Pfand verfallenen Weingartens (deutsch mit hebräischer Unterschrift) wurde zu einem Bucheinband zurechtgeschnitten und dürfte den Falt- und Gebrauchsspuren nach zu schließen auch als solcher verwendet worden sein. Noch in situ vgl. z. B. Vorau, Stiftsarchiv, Codex 217: auf dem vorderen Innendeckel des Codex klebt als Deckblatt eine zurechtgeschnittene Schuldurkunde des Wiener Neustädter Bürgers Heinrich Luchs und seiner Frau Anna bei dem Juden Mayerlein, Sohn des Sluemlein, aus dem Jahr 1385.

19 Vgl. die Datenbank des laufenden Projekts „Hebräische Handschriften und Fragmente in österreichischen Bibliotheken“ (URL http://hebraica.at/; einges. 7.6.2014). 
Inhalts zur Verfügung standen. So verlangte nach dem Tod des prominenten Wiener Juden Lebman 1314 einer von dessen adeligen Schuldnern, Rudolf von Sachsengang, von Lebmans Witwe Weichsel die Herausgabe seiner angeblich bereits bezahlten Schuldurkunden. Weichsel und ihre Kinder waren in Lebmans Geschäftstätigkeit offenbar nicht eingebunden und konnten die entsprechenden Urkunden zunächst nicht finden; außerdem wussten sie nicht genau, welche Schuldbriefe Rudolf von Sachsengang tatsächlich bereits bezahlt hatte. Es erscheint ungewöhnlich, dass die entsprechenden Urkunden dem Sachsenganger bei der Begleichung der Schulden nicht ausgehändigt worden wären, jedoch konnten Weichsel und ihre Familie mangels schriftlicher Beweise Rudolfs Behauptung, bereits bezahlt zu haben, nichts entgegensetzen. Letztendlich musste der Sachsenganger nur eine Schuld von acht Mark begleichen, über die sich eine Schuldurkunde finden ließ. Gleichzeitig akzeptierte Lebmans Familie Rudolfs Erklärung, dass er für die verlorenen Schuldbriefe bereits achteinhalb Mark bezahlt hatte. Für den Fall, dass die Urkunden doch noch auftauchen sollten und die darin verzeichnete Schuldsumme achteinhalb Mark überstieg, wurde festgelegt, dass zwei (christliche) Schiedsrichter über die Sache entscheiden sollten. Allerdings war diese Regelung auf ein Jahr begrenzt; alle danach noch aufgefundenen Urkunden über Schulden Rudolfs bei Lebman sollten ungültig sein. Interessant ist in diesem Zusammenhang nicht nur die Tatsache, dass Lebmans Familie entgegen den Gepflogenheiten der meisten jüdischen Geschäftsleute nicht an seiner Tätigkeit beteiligt war; die Urkunde erlaubt auch einen Einblick in den physischen Umgang mit Geschäftsurkunden und die praktischen Probleme, die dieser mit sich bringen konnte. ${ }^{20}$

Wir wissen nur wenig über die Aufbewahrung der Urkunden während der Laufzeit des Geschäfts. Besonders die Spitzengruppe der jüdischen Financiers muss eine bedeutende Anzahl von Schuldbriefen in ihrem Besitz gehabt haben, und man möchte annehmen, dass diese aufgrund ihres Wertes im Normalfall auch sicher verwahrt wurden - im Falle Lebmans vielleicht sogar zu sicher, so dass nicht einmal seine Frau sie finden konnte.

Einen Hinweis darauf, wie jüdische Geldleiher ihre Schuldverschreibungen organisierten, geben die meist auf der Plica oder auf der Rückseite - in seltenen Fällen

20 St. Pölten, Niederösterreichisches Landesarchiv, Urkunden Ständisches Archiv, Nr. 89. Regest bei: BRUGger u. WiedL (Anm. 11), S. 188f., Nr. 193. Vgl. Eveline BRUgGER, Adel und Juden im mittelalterlichen Niederösterreich. Die Beziehungen niederösterreichischer Adelsfamilien zur jüdischen Führungsschicht von den Anfängen bis zur Pulkauer Verfolgung (Studien und Forschungen aus dem Niederösterreichischen Institut für Landeskunde 38), St. Pölten 2004, S. 84; DIES., Loans of the Father: Business Succession in Families of Jewish Moneylenders in Late Medieval Austria, in: Finn-Einar ELIASSEN u. Katalin Szende (Hgg.), Generations in Towns. Succession and Success in Pre-Industrial Urban Societies, Newcastle upon Tyne 2009, S. 112-129. Zu Lebmans Familie vgl. Klaus LoHRmann, Die Wiener Juden im Mittelalter, Berlin, Wien 2000, S. 127-130. 
auch auf den Presseln ${ }^{21}$ - der Urkunde angebrachten knappen hebräischen Vermerke, die eine Kurzfassung des Rechtsinhalts (oft nur den Namen des Schuldners und die Schuldsumme bzw. das Fälligkeitsdatum) enthielten. ${ }^{22}$ Im Gegensatz zu hebräischen Unterschriften, auf die noch zurückzukommen sein wird, handelte es sich dabei um Vermerke für den internen Gebrauch, die keine Rechtswirksamkeit besaßen, sondern wohl das rasche Auffinden der richtigen Urkunde erleichtern sollten. ${ }^{23}$

Trotzdem kam es vor, dass Urkunden verloren gingen. Ein solcher Verlust zog Rechtsunsicherheit nach sich, weshalb zur Absicherung gelegentlich eine höhere Instanz eingeschaltet wurde. Als der Jude Isserl aus Neunkirchen im Jahr 1372 den Schuldbrief Haimos von Geroldsdorf verlor, wandte er sich - wahrscheinlich auf Wunsch des Schuldners - an Herzog Albrecht III. um Hilfe. Der Herzog kam dem Ersuchen nach und bestätigte Isserls Erklärung, dass Haimo die Schuld samt Zinsen zurückgezahlt hatte und dass er, Isserl, die entsprechende Schuldurkunde verloren habe und sie deshalb dem ehemaligen Schuldner nicht ordnungsgemäß zurückgeben könne. Daher erklärte der Herzog die nicht auffindbare Urkunde für ungültig; im Fall des Wiederauftauchens sollte sie dem Juden und dessen Erben keinen Nutzen, Haimo und dessen Erben aber keinen Schaden bringen. ${ }^{24}$

Ähnlich gelagert war der Fall einer weiteren jüdischen Witwe, Süßel aus Pressburg (Bratislava), deren verstorbener Ehemann Merchlein aus einer Kremser Familie stammte und auch in Pressburg geschäftlich tätig war. Süßel erschien 1386 als Vertreterin ihres minderjährigen Sohnes vor dem Richter und dem Rat der Stadt Pressburg und gab dort den Verlust einer Urkunde bekannt, die eine Schuld des Pressburger Bürgers Dankhart vor dem Lorenzertor in der Höhe von 36 Pfund weniger 50 Pfennig bei Süßels verstorbenem Mann dokumentierte und mit dem kleinen Siegel der Stadt Pressburg beglaubigt war. Im Gegensatz zur Witwe des oben erwähnten Lebman wusste Süßel also über den Inhalt der Urkunde Bescheid und bestätigte vor dem Stadtrat, dass Dankhart ihr die Schuld samt Zinsen zurückgezahlt habe. Auch hier wurde die verlorene Urkunde für den Fall, dass sie doch noch auftauchte, vorsorglich

21 Vgl. z. B. St. Pölten, Niederösterreichisches Landesarchiv, Urkunden Ständisches Archiv, Nr. 1126, 1165.

22 Vgl. ebd., Nr. 659, Regest bei: BrugGeR u. WiedL (Anm. 1), S. 322f, Nr. 1116: Albero von Streitwiesen und Albero Stuchs stellen dem Juden Heblein aus Lengbach und dessen Frau Roslein einen Schuldbrief über 24 Pfund Wiener Pfennig aus, die am kommenden Faschingstag fällig sind. Auf der Plica steht der hebräische Vermerk „Alber Streitbiser und Alber Stichs 24 Pfund auf Vaschank 126 nach der kleinen Zeitrechnung“ (Übersetzung: Martha KEIL).

23 Für Österreich sind bisher keine von Juden selbst angelegten Geschäftsbücher bekannt, wie sie in anderen Regionen existieren. Vgl. z. B. Annegret Holtmann, Jüdische Geldleihe im Spiegel mittelalterlicher Geschäftsbücher: Das Beispiel Vesoul, in: CLusE (Anm. 13), S. 333-343.

24 Klosterneuburg, Stiftsarchiv, Urkunde 1372 XII 9. Regest bei: Christian LACKNER, Regesta Habsburgica. Regesten der Grafen von Habsburg und der Herzoge von Österreich aus dem Hause Habsburg, V. Abteilung: Die Regesten der Herzoge von Österreich (1365-1395), 2. Teilbd. (1371-1375), Wien, München 2010, S. 111, Nr. 949. 
für ungültig erklärt, und zwar gleichgültig vor welchem Gericht sie vorgelegt wurde ein Zusatz, der wohl der Situation im Grenzraum zwischen dem Herzogtum Österreich und dem Königreich Ungarn geschuldet war. Die Urkunde, die der Pressburger Stadtrat über Süßels Erklärung ausstellte, war Dankhart allerdings noch nicht Absicherung genug, denn er ließ ihren Inhalt zwei Jahre später auch noch durch pezzer sicherhayt willen, wer ob derselb prief verloren wurd in ein Pressburger Stadtbuch eintragen. Diesem Eintrag ist die Überlieferung der Urkunde zu verdanken, denn das Original ging in der Folge tatsächlich verloren. ${ }^{25}$

Natürlich gingen auch die christlichen Geschäftspartner jüdischer Geldgeber nicht immer sorgfältig mit den Urkunden um. 1378 bestätigte der Jude Nachim aus Windischgrätz, dass eine Urkunde über ein Darlehen von 20 Pfund Wiener Pfennig, die sein Schuldner Hugo von Duino ihm geschickt hatte, nicht bei ihm eingetroffen war. Damit kein Unbefugter mit der Urkunde Forderungen erheben konnte, erklärte Nachim sie - auf Deutsch mit hebräischer Beglaubigung - für ungültig. Neben der Absicherung des Gläubigers lag es auch in Nachims eigenem Interesse, sicherzustellen, dass niemand in seinem Namen die Schuld eintreiben konnte und zugleich festzuhalten, dass der Verlust der Urkunde nicht an ihm lag. ${ }^{26}$

Das Konfliktpotential, das sich aus mangelhaft dokumentierten Darlehensgeschäften ergab, war gemeinsam mit dem Wunsch nach verstärkter Kontrolle des jüdischen Geldgeschäfts ein Faktor in der Anlage städtischer Verzeichnisse der jüdischen Schuldbriefe, aus denen sich die eingangs erwähnten Judenbücher entwickelten. Die erste bekannte Maßnahme dieser Art in Österreich ist eine Bestimmung im Stadtrecht von St. Pölten, das Bischof Albrecht II. von Passau 1338 ausstellte. Sie schrieb den Juden der Stadt vor, ihre Schuldurkunden und Pfänder regelmäßig dem Richter zur Bestätigung vorzuweisen, widrigenfalls würden sie ihre Ansprüche verlieren. ${ }^{27}$ Nur zwei Jahre später ließ der österreichische Herzog Albrecht II. ein Judenbuch anlegen, von dem leider nur eine Kopie der lateinischen Einleitung erhalten ist. Diese ist von einem der beiden mit der Anlage betrauten Notare abgefasst und erklärt in äußerst feindseliger, theologisch untermauerter Rhetorik, dass der Herzog ,aufgrund des großen Schadens und der damit verbundenen Streitigkeiten, die aus der Niedertracht der Juden heraus häufig über die Fälschung von Siegeln und Urkunden vorkamen“, zur künftigen Vermeidung dieses Übels im Interesse seiner christlichen und jüdischen Untertanen zwei öffentliche Notare beauftragt habe, alle den Juden versetzten Schuldbriefe in einem Register aufzuschreiben. ${ }^{28}$ Solch unverhohlene

25 Monumenta Hungariae Judaica, Bd. 4: 1371-1564, hrsg. v. Franz Kováts, Budapest 1938, S. 15f., Nr. 9.

26 Wien, Haus-, Hof- und Staatsarchiv, Allgemeine Urkundenreihe 1378 V 4.

27 St. Pölten, Stadtarchiv, Stadtbuch von St. Pölten, fol. 10r-14r. Druck der Judenpassagen bei: BRUGGER u. WIEDL (Anm. 11), S. 341, Nr. 444.

28 Wien, Haus-, Hof- und Staatsarchiv, Allgemeine Urkundenreihe 1340 VI 4. Regest bei: BRUGGER u. WiEDL (Anm. 1), S. 19f., Nr. 476. 
Judenfeindschaft überrascht im Umfeld Albrechts II., der erst kurz zuvor von einem geistlichen Chronisten wegen seines Eintretens für den Judenschutz als fautor Iudeorum verunglimpft worden war; ${ }^{29}$ auch dürfte die Anlage eines herzoglichen ,Zentralregisters' für jüdische Schuldbriefe über das Versuchsstadium nicht hinausgekommen sein. ${ }^{30}$

Der erhobene Vorwurf der jüdischen Siegel- und Urkundenfälschung tritt sonst weder in den überlieferten Urkunden noch in der Historiographie dieses Zeitraums auf; im Lauf des 14. Jahrhunderts lässt sich aber allgemein die Tendenz feststellen, jüdische Kreditgeschäfte immer stärker rechtlich abzusichern. Dies könnte als Hinweis auf eine schwindende Rechtssicherheit der jüdischen Geschäftstätigkeit interpretiert werden, ${ }^{31}$ entspricht allerdings auch einem generellen Trend, der über Geschäfte mit jüdischer Beteiligung hinausgeht und sich genauso bei christlichen Geschäftsabschlüssen findet. ${ }^{32}$ Gerade der Frage, wie mit schriftlichen Belegen umzugehen war, kam dabei große Bedeutung zu. Besonders deutlich zeigt sich dies z. B. am Bestand der Urkunden des Stiftsarchivs Klosterneuburg aus der zweiten Hälfte des 14. Jahrhunderts, die im Rahmen der üblichen Schutz- und Schirmformel für getätigte Geschäfte auch den Einsatz der entsprechenden Urkunden ausdrücklich regeln. Nach österreichischem Landrecht hatte bei einem Güterverkauf der Verkäufer den Käufer gegen alle von dritter Seite auf das Kaufgut erhobenen Ansprüche zu schützen; im Klosterneuburger Umfeld wurde es nun üblich, beim Ausstellen einer Verkaufsurkunde festzulegen, dass die Käufer den Verkäufern im Falle solcher Ansprüche den Kaufbrief und alle vorangegangenen Urkunden leihweise zurückgeben sollten, damit diese sie als Beweis zur Abwehr der erhobenen Ansprüche verwenden konnten. Dies war für Juden im Kreditgeschäft insofern wichtig, als sie häufig verfallene Grundstückspfänder weiterverkauften und genauso wie ein christlicher Verkäufer den Schirm dafür zu übernehmen hatten.

Ein konkretes Beispiel aus dem Jahr 1373: Der Wiener Jude Meister Tenichel beurkundete den Verkauf eines Weingartens in Klosterneuburg, der ihm als Pfand für eine nicht zurückgezahlte Schuld verfallen war. Tenichel besaß eine Urkunde

29 Kalendarium Zwetlense a. 1243-1458, hrsg. v. Wilhelm WatTEnBACH, in: Monumenta Germaniae Historica. Scriptores in folio 9, hrsg. v. Georg Heinrich PeRTz, Leipzig 1925, S. 689-698, hier S. 692. Vgl. Eveline BRUgGer, Minem herren dem hertzogen sein juden - die Beziehung der Habsburger zu „ihren“ Juden im spätmittelalterlichen Österreich, in: 25. Österreichischer Historikertag St. Pölten 2008. Tagungsbericht (Veröffentlichungen des Verbands Österreichischer Historiker und Geschichtsvereine 34), St. Pölten 2010, S. 742-749, hier S. 746f.

30 Lohrmann (Anm. 3), S. 157f.

31 Bezeichnend ist in dieser Hinsicht das ab der Mitte des 14. Jahrhunderts regelmäßig auftretende formelhafte Versprechen des Urkundenausstellers an den jüdischen Gläubiger, die Schuld selbst zu begleichen und weder an eine höhere Instanz abzutreten noch sich bei dieser um einen Tötbrief zu bemühen; s. BRUGGER (Anm. 13), S. 339f.

32 ZehetMAYer (Anm. 5), S. 270f. 
über die ursprüngliche Schuld und die dafür erfolgte Verpfändung des Weingartens; dieser war nach einem entsprechenden Gerichtsbeschluss, über den dem Juden ebenfalls eine Urkunde ausgestellt worden war, in seinen Besitz übergegangen. ${ }^{33}$ Diesen Weingarten verkaufte er in der Folge an den Schaffer des Klosterneuburger Dechants. Tenichel übernahm nach Bergrechts- und österreichischem Landrecht den Schirm für den Weingarten; wenn es zu Ansprüchen von dritter Seite käme, sollte der Käufer ihm die Schuld- und die Gerichtsurkunde leihweise zurückgeben, damit er den Käufer gegen die Ansprüche schirmen könne. Würde ihm der Käufer die Urkunden aber nach entsprechender Aufforderung nicht überlassen, wäre Tenichel der Schirmverpflichtung ledig. ${ }^{34}$

Diese Vorgehensweise unterscheidet sich nicht von der, die bei einem christlichen Verkäufer zum Einsatz gekommen wäre. Ein zeitnaher Fall aus demselben Umfeld, an dem der jüdische Geschäftspartner nur am Rande beteiligt war, zeigt dies deutlich:

Der Klosterneuburger Bürger Wisent auf dem Anger und seine Frau beurkundeten 1379 den Verkauf eines Weingartens und hielten ausdrücklich fest, dass sie dem Käufer die Urkunden zu dem Weingarten übergeben hatten. Es handelte sich dabei erstens um einen Gerichtsbrief, der dem Juden Mosche den genannten Weingarten für eine Geldschuld zusprach, zweitens um den Kaufbrief, mit dem Wisent den Weingarten von Mosche gekauft hatte und drittens um eine hebräische Urkunde mit dem Siegel des Rabbiners (ain juedisch prief mit seins maister insigel), die den Verzicht Mosches auf alle weiteren Rechte an dem Weingarten bestätigte. Falls in Zukunft Rechtsansprüche von dritter Seite auf den Weingarten gestellt würden, sollte der Käufer den Verkäufern die Urkunden zurückgeben, damit diese ihn mit diesen Urkunden und dem Kaufbrief nach österreichischem Landrecht gegen die Ansprüche schirmen konnten. Sollten die Urkunden verloren gehen oder beschädigt (zeprochen) werden, wären Wisent und seine Frau nicht zum Schirm verpflichtet. ${ }^{35}$

Hier wurde also ausdrücklich für den Fall vorgesorgt, dass die zur Beweisführung nötigen Urkunden nicht mehr vorhanden waren - ein Problem, das Christen wie Juden gleichermaßen betreffen konnte. Der Ausdruck zeprochen scheint zunächst auf eine Beschädigung oder Zerstörung des Siegels hinzudeuten. Allerdings war mit dem im Text genannten insigel des Rabbiners mit Sicherheit eine Unterschrift gemeint, ${ }^{36}$

33 Klosterneuburg, Stiftsarchiv, Urkunde 1372 III 2.

34 Klosterneuburg, Stiftsarchiv, Urkunde 1373 VIII 10. Gedruckt bei: Hartmann ZEIBIG, Urkundenbuch des Stiftes Klosterneuburg bis zum Ende des vierzehnten Jahrhunderts, Teil 1 (Fontes Rerum Austriacarum II/10), Wien 1857, S. 453f., Nr. 465 (nach einer kopialen Klosterneuburger Überlieferung, in der - wohl aufgrund fehlender Sprachkenntnisse des Kopisten - die hebräische Unterschrift Tenichels fehlt, die auf der Originalurkunde unter dem deutschsprachigen Text steht).

35 Klosterneuburg, Stiftsarchiv, Urkunde 1379 I 5. Regest bei: ZEIBIG (Anm. 34), S. 477.

36 Der hebräische Begriff chatam, mit dem in hebräischen Urkunden die Unterschrift angekündigt wird, kann sowohl „unterschreiben“ als auch „,siegeln“ bedeuten, vgl. Martha KEIL, Ein Regensburger Judensiegel des 13. Jahrhunderts. Zur Interpretation des Siegels des Peter bar Mosche haLevi, in: 
sodass man insgesamt von einer weiter gefassten Bedeutung des Wortes im Sinn von ,Beschädigung der Urkunde‘ ausgehen muss. Gleichzeitig ist hier deutlich dokumentiert, dass die Rechtsgültigkeit hebräischer Dokumente vom christlichen Geschäftspartner nicht in Frage gestellt wurde.

Dem Siegel im eigentlichen Sinn kam gerade bei der Frage der fortgesetzten Gültigkeit von Urkunden dennoch große Bedeutung zu. Jüdische Siegel stellen eine Seltenheit dar, weil sie nicht notwendig waren: die gängige Beglaubigung für Juden (und auch Jüdinnen) war im Gegensatz zu den häufig illiteraten Christen eben die eigenhändige hebräische Unterschrift, die auch von Christen als legitime Beglaubigung akzeptiert wurde. ${ }^{37}$ Zwar führten manche jüdische Financiers im Geschäftsverkehr mit christlichen Kunden ein Siegel, um ihrem hohen Sozialprestige Ausdruck zu verleihen, allerdings war dies eher die Ausnahme. ${ }^{38}$

Für Christen stellte das Siegel hingegen die wichtigste Form der Beglaubigung dar. Die Beglaubigung durch Zeugenreihen verlor in Österreich im Lauf des 13. Jahrhunderts immer mehr an Bedeutung; ${ }^{39}$ an ihre Stelle traten in Privaturkunden die Siegelzeugen, die zusätzlich zum Aussteller (bzw. bei Siegelkarenz des Ausstellers an

Aschkenas 1 (1991), S. 135-150, hier S. 136. Hebräische Unterschriften werden sogar in deutschsprachigen Urkunden jüdischer Aussteller gelegentlich explizit als „Siegel“ angekündigt, vgl. z. B. Graz, Steiermärkisches Landesarchiv, Allgemeine Urkundenreihe 3497b (1384 August 28): Die Corroboratio der von dem Voitsberger Juden Smoiel und dessen Frau ausgestellten Urkunde kündigt neben dem Siegel des Voitsberger Judenrichters auch das iudisch insigel Smoiels an. Die Urkunde trug erkennbar nur ein einziges (nicht erhaltenes) Siegel, dafür steht unter dem deutschsprachigen Textblock Smoiels hebräische Unterschrift. In den Urkunden christlicher Aussteller tritt diese Gleichsetzung normalerweise nicht auf, allerdings ist davon auszugehen, dass Wisent auf dem Anger, der Aussteller der oben zitierten Urkunde, für den Inhalt der von ihm angeführten hebräischen Urkunde auf einen jüdischen Übersetzer angewiesen war (vgl. Anm. 34) und daher vielleicht dessen Diktion übernahm. Da das Fehlen eines Siegels im eigentlichen Sinn an der hebräischen Urkunde für Wisent klar erkennbar gewesen sein muss, stellte die begriffliche Gleichsetzung für ihn offensichtlich kein Problem dar.

37 Martha KeIL, „Petachja, genannt Zecherl“: Namen und Beinamen von Juden im deutschen Sprachraum des Spätmittelalters, in: Reinhard HäRTEL (Hg.), Personennamen und Identität. Namengebung und Namengebrauch als Anzeiger individueller Bestimmung und gruppenbezogener Zuordnung (Grazer grundwissenschaftliche Forschungen 3 = Schriftenreihe der Akademie Friesach 2), Graz 1997, S. 119-146, hier S. 138-141.

38 KeIL (Anm. 36), S. 135-140; BRUGGeR u. WiEdL (Anm. 3), S. 294.

39 ZeHETMAYER (Anm. 5), S. 268f. Solange Zeugenreihen in Gebrauch waren, traten darin gelegentlich auch Juden auf, vgl. z. B. München, Bayerisches Hauptstaatsarchiv, Klosterurkunde Aldersbach Nr. 44 (1264 April 29), gedruckt bei BRUGGER u. WiEDL (Anm. 11), S. 56f., Nr. 42. Die Urkunde, die der Dechant und der Judenrichter von Krems gemeinsam ausstellten und besiegelten, enthält die Zeugenreihe Chunradus notarius, Wichardus subdyaconus, Helnwicus in foro, Hainricus sagitarius, Hainricus Trawnchiricher, Chunradus cellerarius, Fridelo iudeus, Schnoman, David, Ruben iudei et alii quam plures. 
dessen Stelle) die Urkunde mit ihrem Siegel bestätigten. ${ }^{40}$ Jüdische Aussteller ließen deutschsprachige Urkunden oft vom christlichen Judenrichter (mit)besiegeln, allerdings kamen auch häufig andere christliche Siegelzeugen zum Einsatz. ${ }^{41}$ Siegel spielten also im Rahmen jüdisch-christlicher Geschäftsabschlüsse auch für die beteiligten Juden, die Wert auf die Absicherung ihrer Dokumente legten, eine wichtige Rolle. Schon die österreichische Judenordnung Herzog Friedrichs II. aus dem Jahr 1244, die die rechtliche Grundlage der jüdischen Ansiedlung im Herzogtum Österreich darstellte, sagte den Juden die Unterstützung des Herzogs beim Eintreiben adeliger Schulden zu, wenn die Juden diese mit Brief und Siegel (per suas literas et sigillum) nachweisen konnten. ${ }^{42}$

Aus diesem Grund betraf der Verruf, also die Ungültigkeitserklärung des verlorenen oder zerstörten Siegels eines christlichen Schuldners, auch dessen jüdische Geschäftspartner und wurde entsprechend öffentlich gemacht. Im Jahr 1341 bestätigte der österreichische Herzog Albrecht II. die Urkunden der Judenmeister von Wien, Krems und Wiener Neustadt über den Verruf des zerbrochenen Siegels des herzoglichen Hofmeisters Ulrich von Pergau, der wahrscheinlich, wie es üblich war, in den Synagogen verkündet worden war. ${ }^{43}$ Wenige Monate zuvor hatte König Karl I. von Ungarn bei der Verleihung eines neuen Siegels an die Stadt Ödenburg (Sopron) den Verruf des alten Stadtsiegels unter anderem auch an die Juden, und zwar der Lage der Stadt entsprechend sowohl an die Juden in Ungarn als auch an diejenigen in Österreich, adressiert. Die Veröffentlichung des Verrufs erlaubte jedem, der mit dem verrufenen Siegel beglaubigte Urkunden besaß, diese vorzuweisen, um sie mit einem neuen, gültigen Siegel bestätigen zu lassen. Wurde dies versäumt, verloren die Urkunden ihre Gültigkeit. ${ }^{44}$ Eine ähnliche Vorgehensweise konnte auch beim Tod eines Schuldners zum Einsatz kommen, und dies trotz der Tatsache, dass in den ent-

40 Eine direkte Involvierung der siegelnden Personen in das besiegelte Rechtsgeschäft musste dabei nicht unbedingt gegeben sein, wie die häufig verwendeten Schadlosformeln belegen, vgl. z. B. St. Pölten, Niederösterreichisches Landesarchiv, Urkunden Ständisches Archiv Nr. 1119: die ouch der sach getzewgen sind mit iren anhangunden insigiln yn an schaden.

41 Vgl. z. B. die in Anm. 36 zitierte Urkunde Smoiels aus Voitsberg. Brugger (Anm. 20), S. 23; zum Judenrichter vgl. LoHrmanN (Anm. 20), S. 46-51; Brugger (Anm. 4), S. 149f.

42 Brugger u. Wiedl (Anm. 11), S. 36, Nr. 25. Vgl. Lohrmann (Anm. 3), S. 78f.

43 Wien, Haus-, Hof- und Staatsarchiv, Urkundenreihe Gschwendt, Nr. 66 (1341 Juli 28). Regest bei: Brugger u. Wiedl (Anm. 1), S. 26, Nr. 489.

44 Irmtraut LINDECK-PozZA, Urkundenbuch des Burgenlandes und der angrenzenden Komitate Wieselburg, Ödenburg und Eisenburg, Bd. 4: Die Urkunden von 1328 bis 1342 mit Nachträgen von 1284 bis 1318, Wien, Köln u. Graz 1985, S. 300, Nr. 470. Vgl. z. B. Wien, Haus-, Hof- und Staatsarchiv, Allgemeine Urkundenreihe 1377 VI 29: Die Urkunde Herzog Albrechts III. über den Verruf des verlorenen Siegels des herzoglichen Hauptmanns ob der Enns hält ausdrücklich fest, dass sich nach der Veröffentlichung des Siegelverrufs in der Landschranne und den Synagogen niemand meldete, der entsprechende Urkunden des Hauptmanns besaß, und erklärt daher alle in Zukunft noch auftauchenden Urkunden, die mit dem verrufenen Siegel beglaubigt waren, für ungültig. 
sprechenden Urkunden fast immer ausdrücklich auch die Erben der Beteiligten mitverpflichtet wurden. ${ }^{45}$

Nicht nur bei friedlichen Transaktionen wie dem erwähnten Weingartenverkauf Wisents auf dem Anger, sondern noch viel mehr bei Rechtsstreitigkeiten kam eventuell vorhandenen Schuld- und Pfandbriefen bzw. den darauf basierenden Rechtsansprüchen jüdischer Gläubiger essentielle Bedeutung zu. Nicht immer ging die Einigung so reibungslos über die Bühne, wie es - zumindest dem Befund der Quelle nach - bei den oben zitierten unauffindbaren Schuldurkunden Lebmans der Fall gewesen sein dürfte. So beurkundete etwa der Bergmeister des Wiener Klarissenklosters 1378 einen Gerichtsentscheid über die Klage des Klosters auf nicht bezahlte Abgaben von einem Weingarten. Der Besitzer des Weingartens hatte diesen offenbar als Pfand für ein jüdisches Darlehen gesetzt, denn der klagende Vertreter des Klosters bot gemäß dem nach Landrecht üblichen Prozedere mit dem Fronboten und dem Wiener „Judenmesner“ (Schammasch) an, dass die Witwe des bereits erwähnten Wiener Juden Meister Tenichel mit ihren Urkunden vor Gericht erscheinen und ihre Ansprüche beweisen solle. Da jedoch weder Tenichels Witwe noch ihre Erben vor Gericht erschienen, wurden ihre Urkunden für ungültig erklärt und der Weingarten für die versessenen Abgaben dem Kloster zugesprochen. ${ }^{46}$ Die Involvierung des „Judenmesners“, dessen Funktion vor Gericht dem des christlichen Fronboten entsprach, ${ }^{47}$ zeigt, dass auf die Interessen der jüdischen Seite Rücksicht genommen werden sollte; warum Tenichels Witwe dem Angebot nicht nachkam und ob es sich hier möglicherweise um einen weiteren Fall von nicht auffindbaren Schuld- bzw. Pfandbriefen handelte, die ihre Sache vor Gericht aussichtslos gemacht hätten, geht aus der Urkunde nicht hervor.

Hinweise auf den Verlust von Schuldurkunden im Zuge von Verfolgungen finden sich im Herzogtum Österreich vor der Vernichtung der jüdischen Ansiedlung 1420/21 (,Wiener Gesera“) nur sehr selten, was wohl zum größten Teil daran liegt, dass die habsburgischen Landesfürsten ihre jüdischen Untertanen lange Zeit vor Verfolgungen zu schützen versuchten ${ }^{48}$ - Verfolgungen, die mit angeblichen Hostienschändungen oder ähnlichen religiösen Motiven begründet wurden, deren Betreiber aber (wie die Wiener Annalen Ende des 14. Jahrhunderts anlässlich einer Judenverfolgung in der Steiermark und in Kärnten vermerkten) geren gotz marter gerochen hieten und daraus ir brief und geltschuld von den unseligen juden ledig gewesen. ${ }^{49}$ Erst im Zuge der

45 Reinhard HÄRTEL, Die Zugehörigkeit des Pittener Gebietes zu Österreich oder Steier im späten Mittelalter, in: Jahrbuch für Landeskunde von Niederösterreich Neue Folge 50/51 (1985), S. 53-134, hier S. 125.

46 Wien, Haus-, Hof- und Staatsarchiv, Allgemeine Urkundenreihe 1378 V 1.

47 KeIL (Anm. 5), S. 50.

48 BRugger (Anm. 29), S. 742-749.

49 Österreichische Chronik von den 95 Herrschaften, hrsg. v. Joseph SEEmüLlER (Monumenta Germaniae Historica, Deutsche Chroniken 6), Hannover 1906-1909, ND München 1980, S. 238. Zu der in der zitierten Stelle erwähnten Verfolgung vgl. BRUGGER (Anm. 4), S. $220 \mathrm{f}$. 
Gesera, die eine radikale Abkehr von der bis dahin praktizierten landesfürstlichen Judenpolitik darstellte, kam es zum systematischen, obrigkeitlich verordneten Raub jüdischen Schriftgutes. ${ }^{50}$ Herzog Albrecht V. zog gemeinsam mit dem Rest des jüdischen Besitzes auch die offenen Schuldforderungen der Juden ein. Im sogenannten Schatzgewölbe-Register aus der Mitte des 16. Jahrhunderts sind die Belege für das den Juden - oft unter Folter - abgepresste Vermögen inventarisiert, auch wenn diese Belege selbst leider verlorengegangen sind. Neben Verzeichnissen des Geldes und der Kleinodien der Juden und einem Schuldenregister für das Jahr 1420 listet das Inventar auch eine Reihe von zusammengebundenen Schriften auf, darin ligen villerlei zetlen und inventari der gedachten Juden gefunden und gezaigten clainat, silbergschmeidt und schulden. ${ }^{51}$ Unter den den Juden geraubten Wertgegenständen hatten sich also auch die noch in ihrem Besitz befindlichen Schuldbriefe befunden; nach dem jiddischen Bericht, der der Verfolgung den Namen gab, erließ der Herzog den Schuldnern der Juden die aufgelaufenen Zinsen und ließ sich das Kapital selbst auszahlen. ${ }^{52}$ Allerdings war es mit dem Entzug der Schuldurkunden nicht getan, wenn von anderer Seite politischer Druck gemacht wurde: der ungarische König Sigismund und der steirische Herzog Ernst erhoben als Herrscher jener Gebiete, in die die vertriebenen österreichischen Juden geflüchtet waren, im Namen ihrer neuen jüdischen Untertanen Entschädigungsansprüche. König Sigismund erklärte in einem Schutzbrief für den Juden Isserl aus Bruck, dass dieser in Österreich seine geldschuld under im gelassen hat czwisschen etlichen cristen [...] durich dez willen ir geldschuldbriffe und ir judenpuch, darynne dieselbig ir geldschuld geschriben stet, im enczogen und genommen worden sein czu grossen scheden. Eine fast gleichlautende Bestätigung erhielt der Jude Haubel aus Hainburg. Beide sollten von den Amtleuten König Sigismunds Hilfe bei der Eintreibung ihrer Außenstände erhalten, obwohl ihnen die schriftlichen Belege geraubt worden waren; allerdings hatten sie die Hälfte bzw. ein Drittel der eingebrachten Summe an den König abzutreten und darüber durich eyner sichrer auzweizung und geczewgnuzz willen eine Quittung auszustellen. ${ }^{53}$ Bei entsprechen-

$50 \mathrm{Zu}$ den während der Gesera geraubten hebräischen Handschriften vgl. KEIL (Anm. 14), S. 59 mit Anm. 40. Allgemein zur Gesera vgl. Arye Maimon, Mordechai Breuer u. Yacov Guggenheim (Hgg.), Germania Judaica III/3: Gebietsartikel, Einleitungsartikel und Indices, Tübingen 2003, S. 1986-1988; Brugger (Anm. 4), S. 221-224; Lohrmann (Anm. 3), S. 298-309; Ders. (Anm. 20), S. 155-173; Martha KeIL, Bet haKnesset, Judenschul. Die mittelalterliche Synagoge als Gotteshaus, Amtsraum und Brennpunkt sozialen Lebens, in: Wiener Jahrbuch für jüdische Geschichte 4 (1999/2000), S. 71-89, hier S. 73f.

51 Arthur Goldmann, Das Judenbuch der Scheffstraße zu Wien (1389-1420), mit einer Schriftprobe (Quellen und Forschungen zur Geschichte der Juden in Deutsch-Österreich 1), Wien, Leipzig 1908, S. 113, Anm. 1.

52 Ebd., S. $125 f$.

53 Monumenta Hungariae Judaica, Bd. 1: 1092-1539, hrsg. v. Ármin Friss u. Mór WeISz, Budapest 1903, S. 150-154, Nr. 114f. Vgl. Lohrmann (Anm. 3), S. 308f. 
dem obrigkeitlichen Interesse bedeutete der Verlust von ,Brief und Siegel` also noch nicht das Ende eines Anspruchs - die dafür zu leistende Zahlung hatte aber selbstverständlich wieder verbrieft zu werden. 